\title{
Integrating RFIDs and Smart Objects into a Unified Internet of Things Architecture
}

\author{
Evangelos A. Kosmatos, Nikolaos D. Tselikas, Anthony C. Boucouvalas \\ Department of Telecommunications Science and Technology, University of Peloponnese, Tripoli, Greece \\ E-mail: \{vkosmat, ntsel, acb\}@uop.gr \\ Received March 31, 2011; revised April 6, 2011; accepted April 12, 2011
}

\begin{abstract}
The term Internet of Things refers to the networked interconnection of objects of diverse nature, such as electronic devices, sensors, but also physical objects and beings as well as virtual data and environments. Although the basic concept of the Internet of Things sounds simple, its application is difficult and, so far, the respective existing architectural models are rather monolithic and are dominated by several limitations. The paper introduces a generic Internet of Things architecture trying to resolve the existing restrictions of current architectural models by integrating both RFID and smart object-based infrastructures, while also exploring a third parameter, i.e. the social potentialities of the Internet of Things building blocks towards shaping the "Social Internet of Things". The proposed architecture is based on a layered lightweight and open middleware solution following the paradigm of Service Oriented Architecture and the Semantic Model Driven Approach, which is realized at both design-time and deployment-time covering the whole service lifecycle for the corresponding services and applications provided.
\end{abstract}

Keywords: Internet of Things, RFID, Smart Objects, Blojects, Service Oriented Architecture, Semantic Model Driven Approach

\section{Introduction}

Over the last decade the term Internet of Things (IoT) has attracted attention by projecting the vision of a global infrastructure of networked physical objects, enabling anytime, anyplace connectivity for anything and not only for anyone. IoT refers to a world where physical objects and beings as well as virtual data and environments, all interact with each other at the same space and time. IoT was initially inspired by members of the RFID development community, who referred to the possibility of discovering information about a tagged object by browsing an internet address or database entry that corresponds to a particular RFID. In this direction, several research activities focus on linking tens of thousands of sensor networks using a convergence of technologies that will allow companies and individuals to keep track of every physical item on earth at every moment. The above as well as several similar studies envisage an IoT architecture, the potentialities of which are inevitably limited by the dichotomy imposed by RFID nature, i.e. the heterogeneity between the plain and passive RFID tags and the networked RFID readers. In order to overcome this re- striction, some researchers are working towards an alternative architectural model for the IoT as a loosely coupled, decentralized system of smart objects-that is, autonomous physical/digital objects augmented with sensing, processing, and network capabilities.

This paper introduces a more generic IoT architecture by integrating both the RFID and smart object-based infrastructures, while also exploring a third parameter, i.e. the social potentialities of IoT building blocks towards shaping the "Social Internet of Things". The present paper is organized as follows; Section 2 outlines the state of the art of current IoT architectural models by analyzing recent studies and research activities as well as by classifying them to the corresponding perspective that the proposed architectural model envisages to integrate. The proposed integrated architectural model is presented in Section 3. Section 4 depicts the corresponding implementation issues. In particular, Section 4 analyzes the layered lightweight and open middleware solution following the paradigm of Service Oriented Architecture, on which the overall architectural framework is based, as well as the Semantic Model Driven Approach, which is realized at both design-time and deployment-time cov- 
ering the whole service lifecycle for the services and applications provided. Section 5 describes an indicative use case scenario emphasizing the inter-scientific and inter-domain potentialities and opportunities emerging by the implementation of the proposed vision for the future IoT, while the paper is summarized in Section 6 with concluding remarks and suggestions for future work.

\section{Internet of Things Architectural Models}

\subsection{RFIDs Perspective}

The Internet of Things (IoT) [1] was initially inspired by members of the RFID development community, who referred to the possibility of discovering information about a tagged object by browsing an internet address or database entry that corresponds to a particular RFID or Near Field Communication (NFC) tag [2]. In this way, real-world everyday objects acquire unique digital identities and can then be integrated into a network as well as be associated with digital information or services. Everyday objects include not only the electronic devices we encounter and use daily and technologically advanced products such as equipment and gadgets, but "things" that we do not normally think of as electronic at allsuch as food, clothing, and furniture; materials, parts, and equipment; merchandize and specialized items; landmarks, monuments and works of art; and all the miscellany of commerce, culture and sophistication.

A very recent survey on the IoT [3] states that unquestionably, the main strength of the IoT idea is the high impact it will have on several aspects of everyday life and behavior of potential users. From the point of view of a private user, the most obvious effects of the IoT introduction will be visible in both working, domestic and entertainment fields. In this context, independent living, enhanced learning and entertainment, e-health and automotive are only a few examples of possible application scenarios in which the new paradigm will play a leading role in the near future. Similarly, from the perspective of business users, the most obvious effects of the IoT deployment will be visible in fields such as, intelligent manufacturing, logistics, retail, supply chain management, product lifecycle management, reliable and safe transportation of people and goods.

The aforementioned potentialities and perspectives of IoT are considered highly challenging by the US National Intelligence Council (NIC), which includes IoT in the list of six "Disruptive Civil Technologies" with potential impacts on US national power [4]. NIC foresees that "by 2025 Internet nodes may reside in everyday things-food packages, furniture, paper documents, and more”. It underlines the major opportunities that will emerge, starting from the attitude that "popular demand combined with technology advances could drive widespread diffusion of an IoT that could, like the present Internet, contribute invaluably to economic development".

In this direction, several research activities focus on linking tens of thousands of sensor networks using a convergence of technologies that will allow companies and individuals to keep track of every physical item on earth at every moment, while addressing the privacy and security concerns. The ASPIRE research project [5] aims at lowering software and integration costs associated with RFID deployment. To that end, ASPIRE is developing a lightweight, programmable, standards-compliant, integrated and privacy-friendly RFID middleware platform, along with a range of tools intended to facilitate RFID deployment. The BRIDGE research project [6] configures a set of research, development and implementation tools to enable the deployment of RFID related applications taking into consideration the technical, social and educational challenges. The Perci framework [7] integrates Web services and Physical Mobile Interaction (PMI) with tagged everyday objects. In [8] the authors developed a suite of Web-based, user-level tools and applications designed to empower users by facilitating their understanding, management and control of personal RFID data.

\subsection{Smart Objects Perspective}

The above and similar studies envisage an IoT architecture the potentialities of which are inevitably limited by the dichotomy imposed by RFID nature - of simple RFID tags and networked RFID readers. In order to overcome this restriction, some researchers [9-11] are working toward an alternative architectural model for the Internet of Things as a loosely coupled, decentralized system of smart objects-that is, autonomous physi$\mathrm{cal} /$ digital objects augmented with sensing, processing, acting and network capabilities. In contrast with passive RFID tags, smart objects carry chunks of application logic that allow them to make sense of their local situation and interact with human users and other nearby smart objects. They sense, log, and interpret what is occurring within themselves and the world, act on their own, intercommunicate with each other, exchange information with people and discover where they are, which other objects are in their vicinity and what happened to them in the past.

The idea of smart objects and the IoT was recently popularized by Sterling [9]. Sterling coined the term spime to describe a new category of space-time, loca- 
tion-aware, environment-aware, self-logging, self-documenting, uniquely identified objects that provide a lot of data about themselves and their environment. According to Sterling, one could track the entire existence of an object, from the time before it was made (its virtual representation), through its manufacture, its ownership history, its physical location, to its eventual obsolescence and breaking-down back into raw material to be used for new instantiations of objects. In [10] authors envisage IoT as a composition of smart objects that can understand and react to their environments. Through practical experimentation and by prototyping some generations of smart objects, authors identified three canonical smart object types: Activity-aware objects, policy-aware objects, and process-aware objects. They also identified the smart-object design space as a space of three dimensions: Awareness, representation and interaction. An architectural framework for building smart object systems is described in [11]. The authors design an artifact framework for representing smart objects and a pervasive application model to leverage the services of smart objects dynamically. In a similar direction, the Hydra middleware [12] allows developers to incorporate heterogeneous physical sensing devices into their applications by offering easy-to-use web service interfaces for controlling any type of physical sensor devices irrespective of its network technology. It also incorporates means for device and service discovery, semantic model driven architecture and security.

\subsection{Social perspective}

All the above approaches focus on the tangible side of IoT and they inevitably have not taken into consideration the social potentialities in the same way that the classic internet was unaware of the Web 2.0 capabilities.

In [13] Julian Bleeker made the first approach in this direction by coining the term blogjects to describe the objects that blog. Blogject is a neologism which is meant to focus attention on the participation of objects and things in the sphere of networked social discourse variously called the blogosphere, or social web. The blogjects are searchable, track their location, use histories, are able to capture information about the happenings in their surroundings, communicate with other informative social beings and disseminate all of that anywhere in the world. The project "Pigeon that Blogs" [14] implemented this approach by using a flock of pigeons equipped with wireless connections to internet, GPS device and an environmental sensor that records the levels of toxins and pollutants in the air through which they fly. They disseminate their flight paths, viewable on a Google Map, together with information about the current toxic state of the local atmosphere. The Pigeon that Blogs, which is a mash-up of GPS, GSM communications technology and pollution sensors, represents a species evolution. It is a pigeon pollution Google Maps mash-up and like all good mash-ups it means more than the sum of its parts.

\section{The Proposed Integrated Architecture}

The proposed IoT Architecture introduces a more generic IoT architecture by integrating both the RFID and smart object-based infrastructures. In this framework, RFID tagged objects will be considered to be objects supporting primitive functionalities, while smart objects, as described in previous studies [10-11], will be objects supporting complex functionalities, resulting in a superset of objects. The necessity of integration between different IoT architectures is imposed by two facts: 1) RFID tags are widespread among all aspects of daily life and furthermore, are cheap and easily produced. Envisaging a future IoT without RFID tags and readers is rather utopian. 2) The research on nanoelectronic devices and polymers electronics is used for developing cheap, nontoxic and even disposable electronic sensors and objects that include logic. This development will enable the production at low cost of smart objects which will surpass their limited predecessors, RFID tags. A high-level approach of the proposed IoT architecture is illustrated in Figure 1.

The innovation of the proposed architecture originates from the initial consideration of IoT integrated vision. The applications developed on an IoT architecture compromised by RFID tags are limited to identification and tracking. The integration of sensing and acting smart objects into passive RFID tags would enable the integration of a lot of completely new applications into the IoT context, spanning from sensor and automation specific applications to combined inter-domain applications. In the latest book of the Cluster of European Research

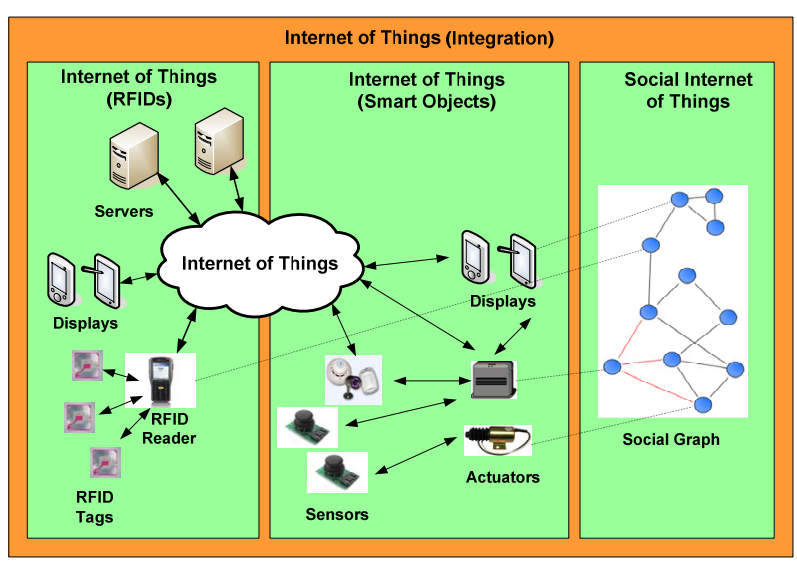

Figure 1. Integrated IoT high-level architecture. 
Projects on IoT, published in 2010 [15], the domains that will be accelerated by the implementation of applications adopting the IoT concept are described in detail. The authors report that the domains that will be highly affected by IoT datum will include: Automotive, intelligent building, telecommunications, healthcare, aerospace, aviation, independent living, pharmaceutical, retail, logistics, supply chain management, product lifecycle management, environmental monitoring, people and good transportation, safety, security, privacy, agriculture and breeding, media and entertainment, insurance and recycling.

In addition, the integration of passive objects (RFID tags) with active objects (smart objects) generates the potentiality of positioning chunks of application logic to selective locations into the IoT framework. The RFIDs architecture approach-limited by its nature-places logic into RFID reader and core components (e.g. Servers on internet). On the other hand, smart objects architecture approach tends to position application logic into local smart objects. The proposed architecture enhances the "Service composition" procedure with the potentiality of preselected or adhoc allocation of application logic into the available building blocks of the IoT architecture. This flexibility allows, firstly, the composition of different versions of the same application according to different requirements and secondly, the formation of several ways of application execution based on different parameter considerations: Connectivity, granularity, resources, energy, mobility, network topology, communication modality, flexibility and quality of service.

Another innovation of the proposed middleware solution is that every object attached to the middleware is considered to have dual nature. On the one hand, it is characterized by its participation to a worldwide infrastructure of networked things. On the other hand, it is described by its social representation. Objects are able to form online communities, participate in one or more social networks and act as blogjects. The proposed architecture enables the creation and management of "social networks of things" composing the "Social Internet of Things". The purposes of the creation may be variant: Creation of a "Grid of Things" with sharable resources to accomplish tasks, creation of a "social network of things" to expand the owners' Web 2.0 social network, creation of a "blogject community of things" which combines their knowledge to search for specific insights and create blog posts, etc.

In this context the proposed architecture is generic enough aiming not only to integrate both architectures, but also to extend the social scope of IoT building blocks from a local level to community, national and global levels respectively. The objects of the final IoT infra- structure will become active participants in the creation, maintenance and knitting together of social formations through: 1) the exploitation of similar characteristics and context parameters, 2) the dissemination of meaningful insights that, until now, were not easily circulated in human readable form.

\section{Implementation Issues}

\subsection{Middleware Solution}

The realization of an integrated IoT architecture can become feasible by the implementation of a lightweight and open middleware solution supporting the aforementioned proposed architectural model. The middleware lies between the heterogeneous objects (RFIDs, smart objects, sensors, actuators) participating in the IoT and the applications using the object capabilities and potentialities. The proposed middleware is a software layer comprising of a set of sub-layers that mediates between the technological and the application levels based on interacting components/modules and abstracting resource and network functions. The architecture of the proposed middleware solution is depicted in Figure 2. The proposed middleware architecture is selected to follow the paradigm of Service Oriented Architecture (SoA) [16-17]. This selection is dictated by two facts: 1) the adoption of the SOA principles allows for decomposing complex and monolithic systems into applications consisting of an ecosystem of simpler and well-defined components. This feature of hiding the details of different technologies exempts the programmer from issues

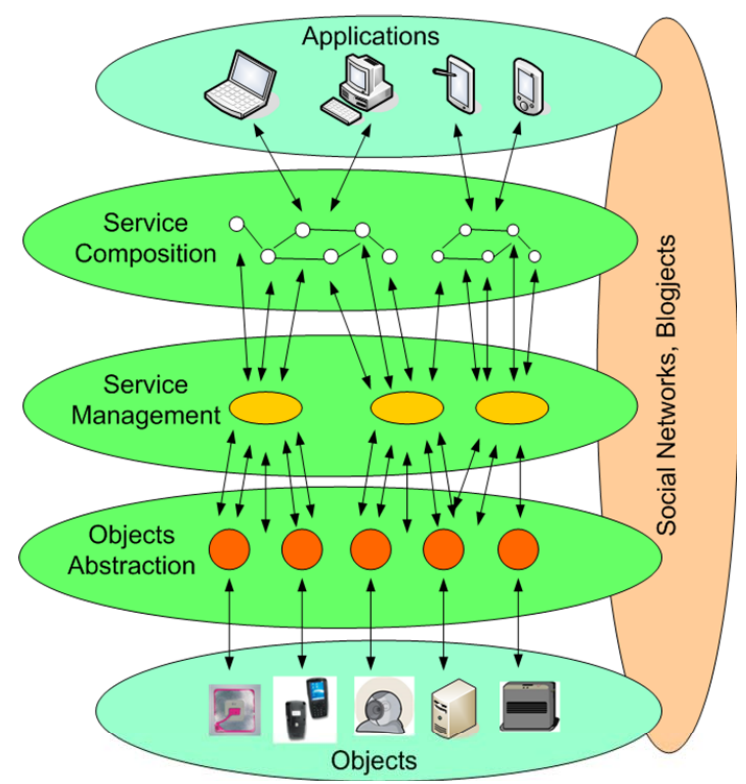

Figure 2. IoT middleware architecture. 
ware architectures proposed in recent years for IoT fol that are not directly pertinent to her/his focus, which is the development of the specific application enabled by the IoT infrastructures. 2) The vast majority of middleware architectures proposed in recent years for IoT follow the SOA approach [3], [14]. Being in accordance with the aforementioned approaches gives the proposed solution the potentiality of a future seamless integration to already implemented solutions. In addition, the selection of an open architecture enables third party developers to integrate their applications making the final solution highly flexible and extensible.

The main goal of the middleware solution is the abstraction of device functionalities and communication capabilities by providing a common set of services and an environment enabling service composition and service orchestration. In this direction, the entire software layer is analysed into a set of sub-layers, as described below.

The "Objects Abstraction" layer is responsible for the translation of the services available to a set of device specific commands and vice versa, following a welldefined notification model. The "Objects Abstraction" layer actually enables the binding of real world objects, i.e. the ones described in Section 2, to the middleware architecture. Through this layer the real world objects provide their capabilities to the upper layers, thus enabling efficient service management, creation and orchestration. This functionality is realized by semantically describing the objects capabilities in such a way that machine agents can understand and use them. In this direction the objects participating in the IoT architecture are semantically described based on ontologies (OWL, OWL 2).

The "Service Management" layer provides a basic but extensible set of functions for the connected objects including dynamic object discovery, status monitoring, mapping of available services to objects and service configuration. The pre-mentioned functionality is actually applied to the corresponding abstract object entities of the underlying layer and is transparently exposed to the overlaying layers, respectively.

The "Service Composition" layer provides the corresponding functionality required for the composition of either plain or more complex services by joining and combining services exposed by the Service Management layer. Service composition is realized in terms of workflows of business processes using workflow languages and web services definition languages (e.g. BPEL, WSDL). The "Service Composition" layer also provides the required orchestration engine used to bind the available interfaces of the corresponding services to the underlying "objects" on which a process will be executed and, finally, it models conditional flow dependency patterns by providing the corresponding required handlers (such as event, data manipulation and transaction handlers).

The "Applications" layer is on top of the architecture. Actually, this layer is not considered to be part of the middleware, but exploits the provided functionalities of the overall middleware architecture.

The vertical "Social Networks, Blojects" layer realizes the notion of sociality in the context of the proposed middleware architecture. As previously mentioned, every object attached to the middleware is considered to have dual nature. The tangible nature of passive or smart objects is depicted by offering their resources and capabilities to the upper layers in order to form services and support applications. The social nature is realized in the middleware architecture by using this layer in order to provide functions for the discovery, creation, management and configuration of social networks and blogject communities.

\subsection{Service Lifecycle}

The proposed solution is following the Semantic Model Driven Architecture (MDA) [18], which differentiates between the physical device and the application view of the device. The Semantic MDA approach introduces the concept of Semantic Devices. Each Semantic Device represents a model of a real device (RFID, smart object, sensor, actuator) and serves as logical unit, which can be semantically discovered and provide information about its capabilities and services. In the proposed middleware the description of Semantic Devices as well as the mapping to the physical devices are realized in the "Objects Abstraction" layer by using device OWL2 [19] ontology. During the design-time more complex semantic devices can be constructed by coupling several semantic devices enhanced with logic definition between them. The high potentiality of semantic MDA originates from its capability of hiding all the underlying device specific complexity of discovering, mapping, and accessing, leaving the developers undisturbed while solving the application problems. In the context of the proposed middleware the Semantic MDA approach is realized at both design-time and deployment-time covering the whole service lifecycle. The service lifecycle is depicted in Figure 3.

The service development can be realized by a service composition tool supported by the middleware infrastructure. By using the service composition tool the developer has two options: Either to create a new service from scratch or to compose a service using pre-existing building blocks of services, which are called service templates.

In the first case the developer is free to design simple or complex services by utilizing a plug-in designed for 


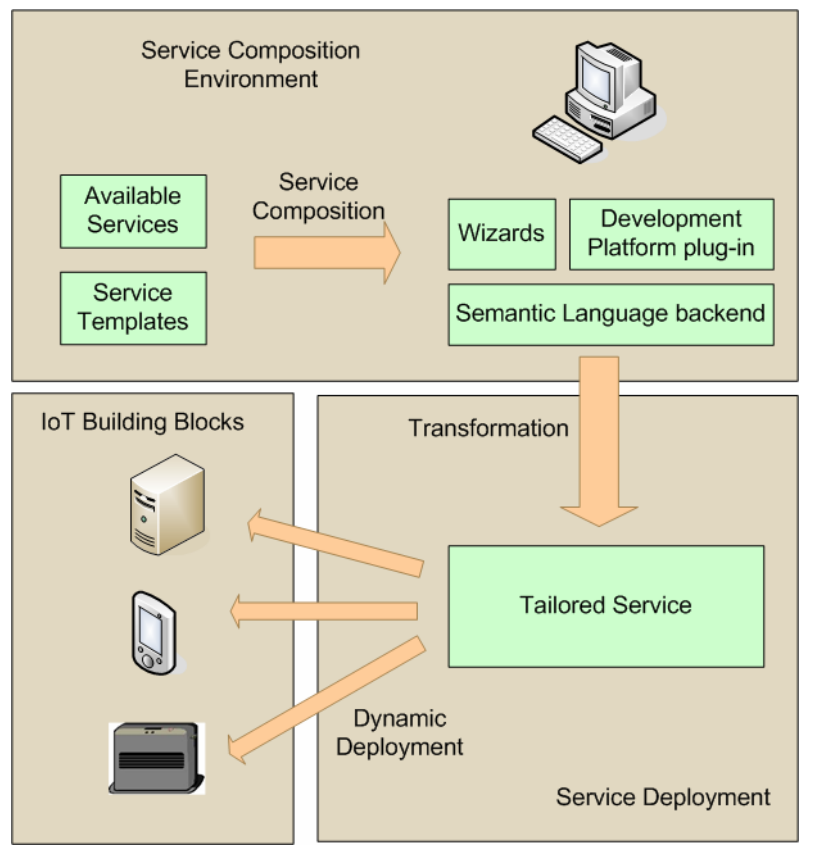

Figure 3. Service lifecycle.

the specific object-oriented language environment, available semantic descriptions of the devices and several interface description files (WSDL). The semantic description of a device is realized by a link to the OWL description of the specific physical device. The WSDL files describe simple services abstracted from device specific services through semantic representation or composed services of already coupled simple services. The developer can import several WSDL files into the language environment and use their capabilities enhanced with additional business logic to implement a variety of services spanning from sensing related services to close loop sensing/actuating services. The developer selects the services to be used among the available set of services in the context of the application under development, while leaving the incorporated devices as generic as possible. The service composition tool generates code for both the service and the devices. The resulting services can be further combined into more complex services forming trees of services and succeeding in archiving a high level of reusability.

The second option, the creation of a service through templates, is suitable for either experienced service developers or inexperienced users. The philosophy, in this case, is that the developer will be able to configure and orchestrate pre-existing building blocks of services into more complex service choreographies based on templates. The service templates is a pool of configurable and easy to use services including 1) common services supported by the majority of devices (e.g. tracking functionality), 2) services common to specific type of devices (e.g. location-determination functionality), and 3) service composition templates. The tool provides a user-friendly Service Creation Wizard that, based on the aforementioned templates, guides the user during every step of the service development process. The Service Creation Wizard hides low-level functionalities and other technicalities from the service developer, while relevant service creation components are presented to the service developer in a user-friendly manner. After the successful execution of a wizard scenario, the corresponding code is generated both on service and on device side.

In runtime all developed services and applications are driven from the Semantic MDA approach. The service composition tool produces new services "tailored" to the developer's requirements. Following the MDA idea, the final step before deployment is to transform the tailored service description into a platform-specific model. During this phase, the proposed middleware enables the preselected or ad-hoc position of application logic into the available building blocks of the IoT architecture. In this direction, the transformation process generates different versions of the same application execution based on different parameters, considerations and possible restrictions of the participated devices (e.g. device resources: Server, smart-object or RFID tag, connectivity). The service deployment on the participating IoT building blocks is shaped by the selected version of service, while during service runtime the transition from one version to another is possible on demand, meeting the needs of new requirements.

In addition, a significant step in the deployment lifecycle is service discovery. When a device is discovered, the device type is looked up in the device ontology and therefore, mapped, if possible, to a specific device model, otherwise to a more generic one. All services or composition of services designed for the specific or generic device type in the device ontology also become active for the newly coming device. In this manner, already developed services could use a device that was designed and built after the service was deployed as long as the device can be classified through the device ontology.

\section{Use Case Scenario}

An indicative use case scenario is presented stressing the inter-scientific and inter-domain potentialities and opportunities emerging by the implementation of the proposed vision for the future IoT: A number of "smart" cars equipped with wireless connection to the internet, a GPS device, an accelerator sensor and an exhaust sensor are moving in the city of Athens. Smart cars moving in the same areas of a street form groups, while their acceleration results determine the traffic condition of the spe- 
cific street. A service using this information shows graphically the traffic conditions of the streets of Athens. Every driver can use this application to schedule his route or use his GPS Navigator application which uses this information to drive him to his destination. Smart cars can interact with other smart objects, like smart traffic lights. The smart traffic lights of Athens form a community, interact with the nearby smart cars and are self-orchestrated to smooth the traffic conditions throughout the city. In this direction, this community can interact with other traffic light communities (e.g. in $\mathrm{Mu}-$ nich) to request the best practices for resolving the traffic jam problem. Smart cars of the same model participate in the social network of the specific model or company, informing the driver for specific mechanical or electrical problems. In addition, the accelerator and exhaust sensor can be combined by the manufacturers to study the engine performance. On a large scale, the measurements from the exhaust sensors can be used by researchers which study environmental conditions and global warming issues. The smart car accelerator sensor can also sense crashes. In case of heavy crashes it can inform the nearest police and emergency forces. The group of smart cars travelling across Athens can also be considered a community of blogjects. A blog post similar to "Eight accidents, two of which very heavy, took place in Thivon Street on the side of the Village Cinemas complex last week" will trigger several actions: 1 ) inform the drivers to be cautious while crossing this dangerous location, 2) persuade the road construction company to search for road impairments in the specific place, 3) stimulate the qualified ministry to place signs in appropriate positions in order to alert the drivers.

\section{Conclusions and Future Work}

In the present study a unified IoT architecture is proposed integrating the two major trends in the area of IoT architectural models, while, in parallel, it exploits the social aspect of participating objects. The RFID approach, despite its simplicity and scalability, is inevitably restricted by the dichotomy of RFID tags and RFID readers. The smart objects approach is applicable only to objects supporting complex functionalities and therefore, limits the scope of IoT. The proposed IoT architectural model introduces a more generic IoT architecture by integrating both the RFID and smart object-based infrastructures. In this framework, RFID tagged objects are considered to be objects supporting primitive functionalities, while smart objects are objects supporting complex functionalities resulting in a superset of objects. In addition, every object attached to the infrastructure is considered to have dual nature. On the one hand, it is charac- terized by its participation to a worldwide infrastructure of networked things, while, on the other hand, it is described by its social representation. In addition, a lightweight and open middleware solution realizing the proposed IoT architecture is described. The proposed middleware solution follows the SOA paradigm enhanced with functionalities like 1) dynamic allocation of application logic into the desirable building blocks (smart objects, RFIDs, servers) and 2) exploitation of objects social capabilities stemming from the "bloject" perspective. The corresponding service lifecycle spanning from design-time to deployment-time is described, while finally, a use case scenario delineates the potentialities of the proposed architecture is presented.

Future work will mainly focus on 1) evaluating the proposed infrastructure in terms of scalability, adaptability and performance maximization, 2) extending the social scope of the proposed architecture by studying the human-object interaction, especially in social network environments.

\section{References}

[1] International Telecommunication Union, “The Internet of Things,” 7th Edition, International Telecommunication Union Internet Reports, 2005.

[2] R. Want, “An Introduction to RFID Technology,” IEEE Pervasive Computing, Vol. 5, No. 1, 2006, pp. 25-33. doi:10.1109/MPRV.2006.2

[3] L. Atzori, A. Iera and G. Morabito, "The Internet of Things: A survey,” Computer Networks, Vol. 54, No. 15, October 2010, pp. 2787-2805. doi:10.1016/j.comnet.2010.05.010

[4] National Intelligence Council, "Six Technologies with Potential Impacts on US Interests Out to 2025,” Conference Report 2008-07, April 2008.

[5] ASPIRE project, 2010. http://www.fp7-aspire.eu

[6] BRIDGE project, 2010. http://www.bridge-project.eu/

[7] G. Broll, E. Rukzio, M. Paolucci, M. Wagner, A. Schmidt and H. Hussmann, "Perci: Pervasive Service Interaction with the Internet of Things," IEEE Internet Computing, Vol. 13, No. 6, 2009, pp. 74-81. doi:10.1109/MIC.2009.120

[8] E. Welbourne, et al., "Building the Internet of Things Using RFID: The RFID Ecosystem Experience," IEEE Internet Computing, Vol. 13, No. 13, 2009, pp. 48-55. doi:10.1109/MIC.2009.52

[9] B. Sterling, "Shaping Things," Massachusetts Institute of Technology Press, Cambridge, 2005.

[10] G. Kortuem, F. Kawsar, D. Fitton and V. Sundramoorthy, "Smart Objects as Building Blocks for the Internet of Things,” IEEE Internet Computing, Vol. 14, No. 1, 2010, pp. 44-51. doi:10.1109/MIC.2009.143

[11] F. Kawsar and T. Nakajima, "A Document Centric 
Framework for Building Distributed Smart Object Systems," 2009 IEEE International Symposium on Object/Component/Service-Oriented Real-Time Distributed Computing,” Tokyo, 17-20 March 2009, pp. 71-79.

[12] Hydra project, 2010. http://www.hydramiddleware.eu

[13] J. Bleecker, "A Manifesto for Networked Objects-Cohabiting with Pigeons, Arphids, and Aibos in the Internet of Things,” blog, 2006.

[14] “Pigeons that Blogs” project, 2010. http://www.beatrizdacosta.net/pigeonblog.php

[15] H. Sundmaeker, P. Guillemin, P. Friess and S. Woelffle, "Vision and Challenges for Realising the Internet of Things," Cluster of European Research Projects on the Internet of Things, European Commision, 2010.

[16] S. D. Deugd, R. Carroll, K. Kelly, B. Millett and J. Ricker, "SODA: Service Oriented Device Architecture,"
IEEE Pervasive Computing, Vol. 5, No. 3, 2006, pp. 9496. doi:10.1109/MPRV.2006.59

[17] P. Spiess, et al., "SOA-Based Integration of the Internet of Things in Enterprise Services," IEEE Proceedings of International Conference on Web Services, Los Angeles, 6-10 July 2009, pp. 968-975.

[18] P. Kostelnik, M. Sarnovsky, J. Hreno, M. Ahlsen, P. Rosengren, P. Kool and M. Axling, "Semantic Devices for Ambien Environment Middleware," The Internet of Things and Services, 1st International Research Workshop, Sophia-Antipolis, 18-19 September 2008.

[19] P. Hitzler, M. Krötzsch, B. Parsia, P. F. Patel-Schneider and Sebastian Rudolph, "OWL 2 Web Ontology Language: Primer,” 2009. http://www.w3.org/TR/owl2-primer/ 